\title{
Atomization Mechanism of Gallium in Graphite Furnace Atomic Absorption Spectrometry
}

\author{
Shoji ImaI, Tsuyoshi Ibe, Toshiyuki Tanaka and Yasuhisa Hayashi \\ Department of Chemistry, Joetsu University of Education, Joetsu, Nïgata 943, Japan
}

\begin{abstract}
The graphite furnace atomic signal of gallium in pure Ar with a pyrolytic graphite (PG) coated tube contained an unresolved double-peak signal. Thermal decomposition of $\mathrm{Ga}_{2} \mathrm{O}(\mathrm{g})$ produced by carbon-catalyzed decomposition of $\mathrm{Ga}_{2} \mathrm{O}_{3}(\mathrm{~s})$ and an on-surface reduction of $\mathrm{Ga}_{2} \mathrm{O}_{3}(\mathrm{~s})$ to $\mathrm{Ga}(\mathrm{g})$ are proposed for the atomization mechanisms giving the first and second signals, respectively. A small peak indicating the vaporization of $\mathrm{Ga}_{2} \mathrm{O}_{3}$ was observed in an old $\mathrm{PG}$ tube after 220 firings. In the presence of an organic matrix, the signal was enhanced due to the occlusion of $\mathrm{Ga}_{2} \mathrm{O}(\mathrm{g})$ in the porous residue of the pyrolyzed matrix. Addition of oxygen produced a peak on the raising part and another peak on the tailing part of the signal observed in pure Ar. The former peak results from a rapid on-surface reduction of the analyte oxide by active sites renewed on the graphite surface, and the latter peak is the same as the small peak in the old PG tube.
\end{abstract}

Keywords Gallium, graphite furnace atomic absorption spectrometry, atomization mechanism, oxygen effect, organic matrix

Gallium is one of the elements exhibiting a large loss of analyte in graphite furnace atomic absorption spectrometry (AAS) $)^{1-4}$, particularly when using a pyrolytic graphite (PG) tube. At temperatures of 470-520 $\mathrm{K}, \mathrm{Ga}_{2} \mathrm{O}_{3}$ is formed by thermal decomposition of $\mathrm{Ga}\left(\mathrm{NO}_{3}\right)_{3} .^{5}$ The melting point of $\mathrm{Ga}_{2} \mathrm{O}_{3}$ is $2010 \mathrm{~K},{ }^{5}$ which is extremely higher than the appearance temperature $\left(T_{\text {app: }} 1520 \mathrm{~K}, 1350 \mathrm{~K}\right)$ of the AAS signal of $\mathrm{Ga}^{6,7}$ Gallium(III) oxide is reduced to $\mathrm{Ga}_{2} \mathrm{O}$ (bp 1000 $\mathrm{K})$ at $870 \mathrm{~K}$ by such reductants as hydrogen and carbon monoxide. ${ }^{5}$ McAllister noted the formation of gaseous $\mathrm{Ga}_{2} \mathrm{O}$ during atomization cycles above $1000 \mathrm{~K}$ by graphite furnace mass spectrometry ${ }^{8}$, in line with a thermochemical prediction that $\mathrm{Ga}_{2} \mathrm{O}(\mathrm{g})$ is the dominant $\mathrm{Ga}$ species in the gas-phase $\mathrm{Ga}-\mathrm{O}-\mathrm{C}$ system at temperatures between 1300 and $1900 \mathrm{~K}^{8}$ They thus attributed the loss of sensitivity to the formation of the $\mathrm{Ga}_{2} \mathrm{O}(\mathrm{g})$. The following two-step atomization mechanism was proposed for platform atomization using a shadow spectral filming technique., 90 The analyte vaporizes as $\mathrm{Ga}_{2} \mathrm{O}(\mathrm{g})$ from the platform and transfers to the wall, and the $\mathrm{Ga}_{2} \mathrm{O}(\mathrm{g})$ is then reduced to monoatomic state of $\mathrm{Ga}$ on the hot graphite surface. Kuga reported that a clear double-peak signal for gallium appears, and that the second peak disappears when using a zirconiumimpregnated graphite tube. ${ }^{11}$

Oxygen is chemically active and is useful as a matrix modifier that promotes ashing in graphite furnace AAS. When oxygen is added to the purge gas, hightemperature shifts in $T_{\text {app }}$ take place for elements atomized via oxide intermediates ${ }^{8,12-14}$, in response to a change in the oxygen partial pressure in the furnace. It was also reported that the oxygen activates the graphite surface. ${ }^{15,16}$ Ascorbic acid and sucrose are matrix modifiers which promote reduction by active carbon. Pyrolysis of ascorbic acid also yields a porous PG layer on the tube wall.

In the present work, a low-temperature shift in an oxygen-Ar mixture and a high-temperature shift in the presence of an organic matrix were observed for gallium which should atomize via dissociation of oxides. This work was undertaken to clarify the effects of oxygen and an organic matrix as well as the atomization mechanism of gallium.

\section{Experimental}

\section{Apparatus}

A Hitachi Model Z-8000 flame and graphite furnace atomic absorption spectrometer equipped with a Zeeman effect background corrector, an optical temperature controller system (Hitachi Model 180-0341) and an automatic data processor were used. Twenty microliters of a sample solution was injected by an automatic sampler. The analytical wavelength and spectral bandwidth were $294.3 \mathrm{~nm}$ and $0.4 \mathrm{~nm}$ for $\mathrm{Ga}$, respectively. An Oki if-800 Model 50 personal computer was used to record the absorbance signal profiles at 20 -ms intervals. The output data from the optical temperature controller were acquired at 4-ms intervals by the computer and stored on a diskette. Temperature data were calibrated using a Chino Model IR-AH1S radiation thermometer. The thermometer was calibrated with a Pt-Rh thermocouple. For this thermometer, the wavelength was $960 \mathrm{~nm}$ and the uncertainties were $0.5 \%$ from 870 to $1500 \mathrm{~K}, 1.0 \%$ from 1500 to $2300 \mathrm{~K}$ and $2.0 \%$ from 2300 to 
Table 1 Standard atomization conditions

\begin{tabular}{lcccc}
\hline \multicolumn{1}{c}{ Stage } & Temp. ${ }^{\mathrm{a}} /{ }^{\circ} \mathrm{C}$ & $\begin{array}{c}\text { Ramp } \\
\text { time } / \mathrm{s}\end{array}$ & $\begin{array}{c}\text { Hold } \\
\text { time/s }\end{array}$ & $\begin{array}{c}\text { Inner gas } \\
\text { flow } / \mathrm{ml} \mathrm{m}^{-1}\end{array}$ \\
\hline Drying & 220 & 50 & 0 & 200 \\
Charring & 900 & 20 & 10 & 200 \\
Atomization $^{\mathrm{b}}$ & 2800 & 0 & 3 & 30 \\
Cleaning $^{2}$ & 2900 & 0 & 3 & 200 \\
\hline
\end{tabular}

a. Programmed for the atomizer unit.

b. The optical temperature controller is used.

$3300 \mathrm{~K}$. The standard atomizer condition is given in Table 1. Each measurement was repeated 5 times.

\section{Reagents}

An aliquot of a commercially available standard solution (Wako Pure Chem. Ind., Ltd.) was properly diluted with water before use. Ascorbic acid and sucrose used were of analytical reagent grade. Distilled and deionized water was purified with a Milli-QII system. Certified gas mixtures of $0.1047,0.2564,0.5106,0.7612$ and $1.009 \%$ oxygen, $1.01 \%$ carbon monoxide in Ar and high-purity Ar (99.995\%) were prepared by Takachiho Chem. Ind.

\section{Results and Discussion}

To elucidate the influence of oxygen, it is important to understand the nature of the graphite tube. The graphite surface comprises crystallites with carbon atoms lying in a basal plane, terminated by carbon atoms in zigzag and armchair configurations. The terminal carbon atoms have unpaired electrons which are available to form bonds with chemisorbed oxygen. The carbon atoms adjacent to the lattice imperfection, present on the surface, have the same reactivity as the terminal carbon atoms. However, the carbon atoms in the basal plane lack this feature, and are essentially nonreactive toward oxygen. ${ }^{17,18}$ The oxidation of graphite is a heterogeneous gas-solid reaction, originating in dissociative chemisorption of oxygen onto specific active sites. An optimum temperature for chemisorption is $850 \mathrm{~K}$, and all of the surface oxygen is desorbed as $\mathrm{CO}$ or $\mathrm{CO}_{2}$ at temperatures above $1250 \mathrm{~K}$, leading to formation of new active sites and decomposition of the basal plane. Sturgeon et al. ${ }^{19}$ reported, by use of a PG tube, that the partial pressure of oxygen in an Ar purge gas decreases at temperatures above $1300 \mathrm{~K}$ according to the reaction

$$
\mathrm{O}_{2}(\mathrm{~g})+2 \mathrm{C}(\mathrm{s}) \longrightarrow 2 \mathrm{CO}(\mathrm{g}) \text {. }
$$

The partial pressure of $\mathrm{CO}$ in an Ar purge gas begins to increase at $1300 \mathrm{~K}{ }^{20}$ The PG layer formed by thermal decomposition of ascorbic acid in a $P G$ tube releases significantly at temperatures above $1700 \mathrm{~K} .{ }^{21}$

Figure 1 presents typical atomic absorption signals for gallium in a PG tube at different heating rates during

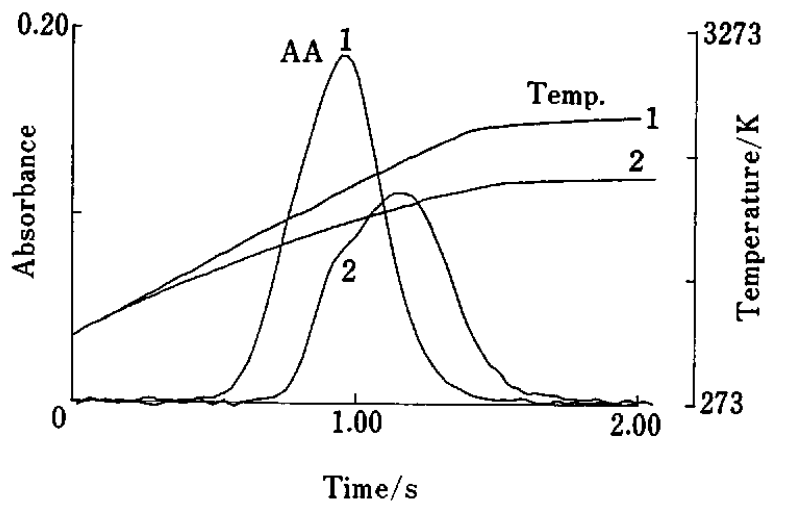

Fig. 1 Atomic absorption (AA) signal for $10 \mathrm{ng}$ gallium with the $P G$ tube and temperature profile (Temp.). Heating rate during atomization: (1) $1150 \mathrm{~K} \mathrm{~s}^{-1}$; (2) $930 \mathrm{~K} \mathrm{~s}^{-1}$.

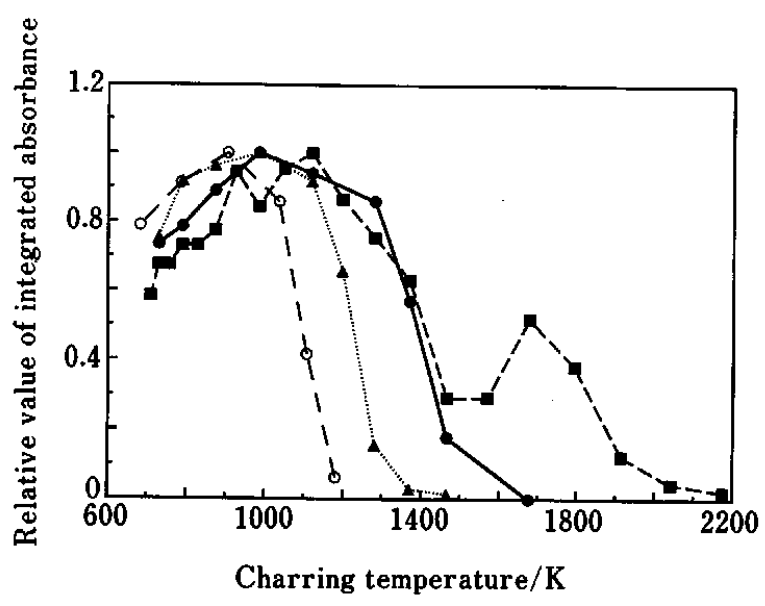

Fig. 2 Effect of charring temperature on the relative value of the integrated absorbance: aqueous solution, PG tube, $10 \mathrm{ng}$ $\mathrm{Ga}(\odot) ; 1 \%$ sucrose, $\mathrm{PG}$ tube, $6 \mathrm{ng} \mathrm{Ga}(\Delta) ; 1 \%$ ascorbic acid, PG tube, $1 \mathrm{ng} \mathrm{Ga}(\square)$; aqueous solution, NPG tube, $4 \mathrm{ng} \mathrm{Ga}$ (O).

atomization. For a heating rate of $1150 \mathrm{~K} \mathrm{~s}^{-1}, T_{\text {app }}$ and the temperature at the maximum absorbance $\left(T_{\max }\right)$ were $1420 \pm 20$ and $1970 \pm 20 \mathrm{~K}$, respectively. When the rate was $930 \mathrm{~K} \mathrm{~s}^{-1}$, a shoulder was observed at $1670-1790 \mathrm{~K}$, showing the gallium signal comprises two unresolved peaks. The earlier and later signals are defined as the first and second signals, respectively.

Figure 2 shows the dependence of the integrated absorbance response on the charring temperature. A significant loss took place at temperatures above $1000 \mathrm{~K}$, since a charring product, $\mathrm{Ga}_{2} \mathrm{O}_{3}$, decomposes to $\mathrm{Ga}_{2} \mathrm{O}(\mathrm{g})$, and this causes substantial sensitivity losses during the atomization cycle. ${ }^{8}$ It was reported that the vapor phase at equilibrium is composed almost entirely of $\mathrm{Ga}_{2} \mathrm{O},{ }^{22}$ i.e., the evaporation follows the scheme

$$
\mathrm{Ga}_{2} \mathrm{O}_{3}(\mathrm{~s}) \longrightarrow \mathrm{Ga}_{2} \mathrm{O}(\mathrm{g})+\mathrm{O}_{2}
$$

The temperatures at $50 \%$ loss are 1420,1230 and $1100 \mathrm{~K}$ 
for an aqueous solution with the PG tube, $1 \%$ sucrose with the $P G$ tube and an aqueous solution with a nonpyrolytic graphite (NPG) tube, respectively. Since the charring of sucrose yields active carbon atoms, and the reduction reactivity of the NPG tube is higher than that of the PG tube, the decrease in the temperature value at $50 \%$ loss indicates that the loss reaction (Eq. (2)) is catalyzed by the active carbon atoms. The following reaction is thus proposed as an additional loss mechanism of gallium:

$$
\mathrm{Ga}_{2} \mathrm{O}_{3}(\mathrm{~s})+2 \mathrm{C}(\mathrm{s}) \longrightarrow \mathrm{Ga}_{2} \mathrm{O}(\mathrm{g})+2 \mathrm{CO},
$$

where $\mathrm{C}(\mathrm{s})$ denotes active carbon. In fact, coating of the PG tube with tungsten led to a ten-fold enhancement of the absorption response with a clear single-peak signal. The single-peak signal $\left(T_{\text {app }}=1370 \pm 10 \mathrm{~K}, T_{\max }=1880 \pm\right.$ $10 \mathrm{~K}$ ) is in a similar temperature range to the shoulder of a signal without the tungsten coating, as shown in Fig. 3. Kuga observed a double-peak signal for gallium when using an NPG tube. ${ }^{11} \mathrm{He}$ also noted that zirconium impregnation of the graphite tube gave a six-fold enhancement of the response with a clear single peak, which is in agreement with the first peak. This supports the occurrence of the carbon reduction by Eq. (3). The dominant gallium species in the gas-phase $\mathrm{Ga}-\mathrm{O}-\mathrm{C}$ system is $\mathrm{Ga}_{2} \mathrm{O}$ at temperatures between 1300 and $1900 \mathrm{~K} .^{8}$ The results mentioned above suggest that the free gallium atoms for the first signal are produced by dissociation of $\mathrm{Ga}_{2} \mathrm{O}(\mathrm{g})$,

$$
\mathrm{Ga}_{2} \mathrm{O}(\mathrm{g}) \longrightarrow 2 \mathrm{Ga}(\mathrm{g})+1 / 2 \mathrm{O}_{2},
$$

which should be promoted by CO. In fact, when a $1.01 \%$ carbon monoxide-Ar mixture was used, the absorbance response was enhanced and a low-temperature shift of $T_{\max }$ was observed with no shift of $T_{\text {app. }}$.

By addition of $1 \%$ sucrose, the absorption response corresponding to the shoulder disappeared, and a clear

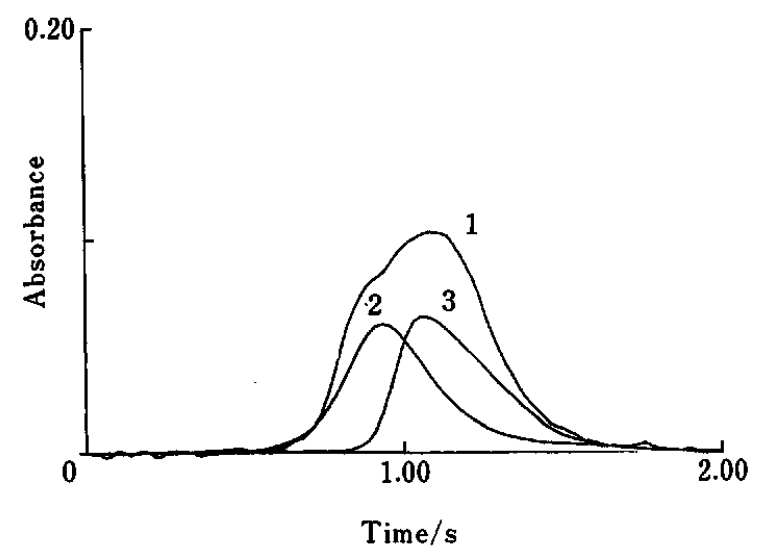

Fig. 3 Atomic absorption signal for gallium. (1) aqueous solution, PG tube, $10 \mathrm{ng} \mathrm{Ga}$; (2) aqueous solution, tungsten coated PG tube, $0.5 \mathrm{ng} \mathrm{Ga}$; (3) 1\% sucrose, PG tube, $1 \mathrm{ng} \mathrm{Ga}$. Heating rate during atomization: $930 \mathrm{~K} \mathrm{~s}^{-1}$. single-peak signal corresponding to the main signal remained, as seen in Fig. 3. A similar signal was observed in $1 \%$ ascorbic acid. The $T_{\text {app }}$ values are $1640 \pm 20$ and $1830 \pm 20 \mathrm{~K}$ respectively for the sucrose and ascorbic acid matrices in the PG tube, and are extremely higher than that for an aqueous solution. At temperatures above $1000 \mathrm{~K}, \mathrm{Ga}_{2} \mathrm{O}_{3}(\mathrm{~s})$ reduces to $\mathrm{Ga}_{2} \mathrm{O}(\mathrm{g})$ according to Eq. (3), and the latter should then be reduced to $\mathrm{Ga}(\mathrm{g})$ by active-carbon atoms formed by a dissociation of pyrolyzed organic matrix at temperatures above $1700 \mathrm{~K}$,

$$
\mathrm{Ga}_{2} \mathrm{O}(\mathrm{g})+\mathrm{C}(\mathrm{s}) \longrightarrow 2 \mathrm{Ga}(\mathrm{g})+\mathrm{CO}
$$

The Arrhenius activation energy $\left(E_{\mathrm{a}}\right)$ for atomization was estimated by the Imai's method. ${ }^{21}$ For the sucrose and the ascorbic acid matrices, the first-order reaction mechanism was chosen in order to make the best possible fits and the $E_{\mathrm{a}}$ values were obtained to be $350 \pm 40$ and $350 \pm 30 \mathrm{~kJ} \mathrm{~mol}^{-1}$, respectively. The enthalpy change for Eq. (5), calculated from reference data ${ }^{23}$, is $680 \mathrm{~kJ} \mathrm{~mol}^{-1}$. Half of this value $\left(340 \mathrm{~kJ} \mathrm{~mol}^{-1}\right)$, namely the enthalpy change for the formation of $1 \mathrm{~mol}$ of $\mathrm{Ga}(\mathrm{g})$, well agrees with the observed $E_{\mathrm{a}}$ value. The process-controlling mechanism corresponds to the gas-solid reaction. Gilmutdinov et al. found, using a platform technique, that a graphite tube wall can reduce $\mathrm{Ga}_{2} \mathrm{O}(\mathrm{g})$ to $\mathrm{Ga}(\mathrm{g})$ at temperatures above $1700 \mathrm{~K} .{ }^{10}$ Inspection of the signals in Fig. 3 suggests that the on-surface reduction could be one of the main sources of free gallium atoms for the second signal.

As mentioned above, the carbon reduction promotes the analyte loss during charring. Since the reduction reactivity of the NPG tube is evidently higher than that of the PG tube, a better sensitivity is expected for the latter. Since the active carbon produced by pyrolysis of organic matrices increases the rate of on-surface reduction (Eq. (3)), the sensitivity value would increase. However, the sensitivity was better for $1 \%$ ascorbic acid and $1 \%$ sucrose, as seen in Table 2. Since the NPG tube has a porous wall, the better sensitivity for the NPG tube would be caused by suppression of the analyte vaporization loss due to its adsorption in the wall. The residue of the pyrolyzed organic matrix also has a porous surface. If the better sensitivity is due only to the

Table 2 Appearance temperature $\left(T_{\text {app }}\right)$, maximum absorption temperature $\left(T_{\max }\right)$ and sensitivity ${ }^{\mathrm{a}}$

\begin{tabular}{llccc}
\hline \multicolumn{1}{c}{ Sample } & Tube & $T_{\text {app }} / \mathrm{K}$ & $T_{\max } / \mathrm{K}$ & Sens./ng \\
\hline Aqueous & PG & $1400 \pm 20$ & $1970 \pm 20$ & 0.31 \\
& NPG & $1450 \pm 30$ & $2260 \pm 20$ & 0.14 \\
$1 \%$ Ascorbic acid & PG & $1830 \pm 20$ & $2090 \pm 10$ & 0.031 \\
& NPG & $1890 \pm 20$ & $2230 \pm 10$ & 0.057 \\
$1 \%$ Sucrose & PG & $1640 \pm 20$ & $1950 \pm 10$ & 0.091 \\
& NPG & $1740 \pm 30$ & $2140 \pm 10$ & 0.064 \\
\hline
\end{tabular}

a. Mass at 0.0044 absorbance of integrated absorbance. 
adsorption of the analyte in the pore of pyrolyzed organic matrix, $T_{\text {app }}$ should be in agreement with that for the NPG tube. However, there is a significant hightemperature shift by the addition of an organic matrix. In the case of ascorbic acid, raising of the charring temperature yields a two-step suppression in the 1150$1450 \mathrm{~K}$ and $1700-2200 \mathrm{~K}$ ranges (Fig. 2). A significant release of a porous residue of pyrolyzed ascorbic acid at temperatures above $1700 \mathrm{~K}$ has been reported. ${ }^{21}$ The second step of suppression is due to occlusion of the analyte in the porous residue of a pyrolyzed organic matrix. The occlusion provides a high-temperature shift of $T_{\text {app }}$ along with disappearance of the first signal. Though sucrose gives a better sensitivity and higher $T_{\text {app }}$, the temperature at $50 \%$ loss during charring is lower than that without sucrose. The analyte loss during charring is increased kinetically due to the much longer time for charring than the appearance time of atomic absorption in the atomization stage.

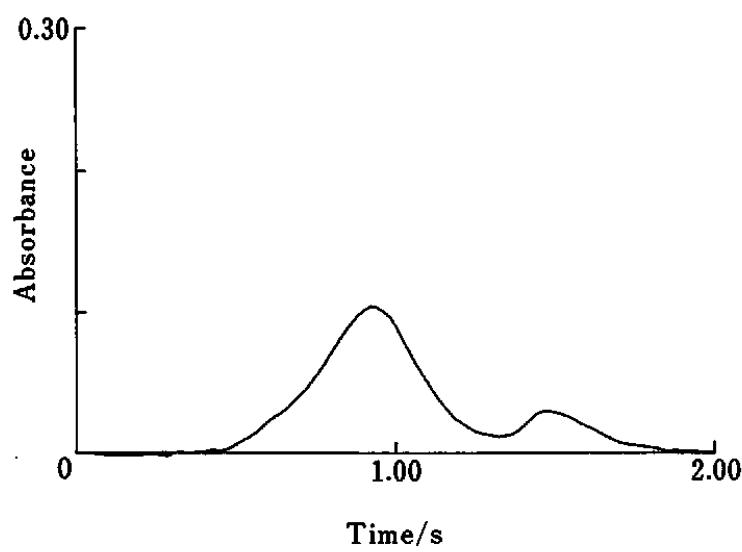

Fig. 4 Atomic absorption signal for gallium with an old PG tube after 220 firings. Aqueous solution, $20 \mathrm{ng} \mathrm{Ga}$.

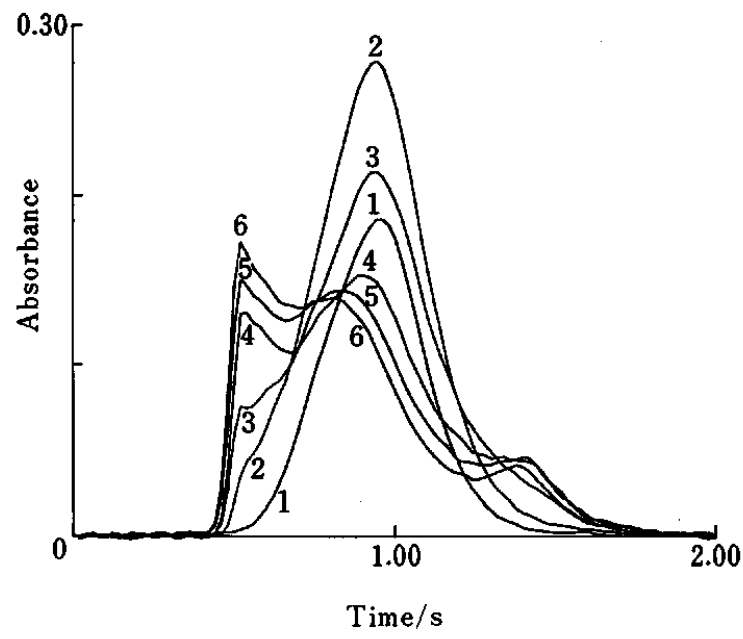

Fig. 5 Variation of atomic absorption signal of $10 \mathrm{ng}$ gallium with the oxygen concentration in the inner Ar gas. Inner gas: high-purity $\mathrm{Ar}(1) ; 0.1047 \% \mathrm{O}_{2}(2) ; 0.2564 \% \mathrm{O}_{2}$ (3); $0.5106 \% \mathrm{O}_{2}(4) ; 0.7612 \% \mathrm{O}_{2}(5) ; 1.009 \% \mathrm{O}_{2}(6)$.
Since an old PG tube after 220 firings under the standard atomization cycle was used, a small peak appeared on the tailing edge of the signal, with $T_{\text {app }}$ and $T_{\max }$ of $2380 \pm 40 \mathrm{~K}$ and $2490 \pm 30 \mathrm{~K}$, as shown in Fig. 4. Repeated firings render the $P G$ tube surface more porous. Because the $T_{\text {app }}$ corresponds to the melting point of $\mathrm{Ga}_{2} \mathrm{O}_{3}(2010 \mathrm{~K})$, the adsorption of $\mathrm{Ga}_{2} \mathrm{O}_{3}$ into the more degraded $\mathrm{PG}$ wall suppresses the formation rate of the monoatomic gallium.

The signal profiles at various oxygen concentrations are presented in Fig. 5. Addition of oxygen elevates its partial pressure in the tube at temperatures below $1900 \mathrm{~K}^{18}$, and the elevated oxygen partial pressure then suppresses the dissociation of the analyte oxides, leading to a high-temperature shift of $T_{\text {app. }}$ The addition of oxygen results in the production of peaks on the rising and tailing parts of the signal in pure Ar. We define the peaks in the order of appearance, as the first, second and third peak. The enhanced absorption response is caused by the suppression of thermal decomposition or reduction of $\mathrm{Ga}_{2} \mathrm{O}_{3}(\mathrm{~s})$. There was an increase in the absorbance at the first and third peaks, and a decrease in that at the second peak, by increasing the oxygen concentration. The $T_{\text {app }}$ value for the first peak decreased with increasing the oxygen concentration and reached a constant value of $1330 \pm 10 \mathrm{~K}$, which is close to the temperature where dissociative oxidation of the graphite structure begins and reproduces active carbon atoms (with an unpaired $\sigma$ electron). This decrease in $T_{\text {app }}$ suggests an on-surface reduction by renewed active sites according to the combination reaction of Eqs. (3) and (5),

$$
\mathrm{Ga}_{2} \mathrm{O}_{3}(\mathrm{~s})+3 \mathrm{C}(\mathrm{s}) \longrightarrow 2 \mathrm{Ga}(\mathrm{g})+3 \mathrm{CO} .
$$

The $T_{\text {app }}$ and $T_{\max }$ values for the third peak are $2330 \pm 40 \mathrm{~K}$ and $2480 \pm 30 \mathrm{~K}$, respectively, and are in agreement with those for the delayed peak observed in the old PG tube after 220 firings. The third peak results from a delayed dissociation of $\mathrm{Ga}_{2} \mathrm{O}_{3}$ adsorbed into the degraded $\mathrm{PG}$ wall.

By raising the charring temperature, the graphite surface oxidation proceeds more effectively, and hence the number of renewed active sites increases. Figure 6 shows the effect of the charring temperature on the signal shape by use of a $0.5106 \%$ oxygen mixture and the standard atomization conditions. The $T_{\text {app }}$ was lowered by raising the charring temperature with a minimum $T_{\text {app }}$ value at $830 \mathrm{~K}$, which is close to the optimum temperature for the oxygen chemisorption on graphite. ${ }^{6}$ The height of the first peak increased with increasing the charring temperature, while that of the second peak decreased. These results indicate that a fraction of gallium atomized by the on-surface reduction ( Eq. (6)) is increased with increasing the extent of oxidation of the tube wall during charring, through an increase in the number of active sites.

Since an increase in the purge gas flow rate causes an increase in the rate of graphite oxidation, leading to reproduction of active sites ${ }^{19}$, an enhancement of the 


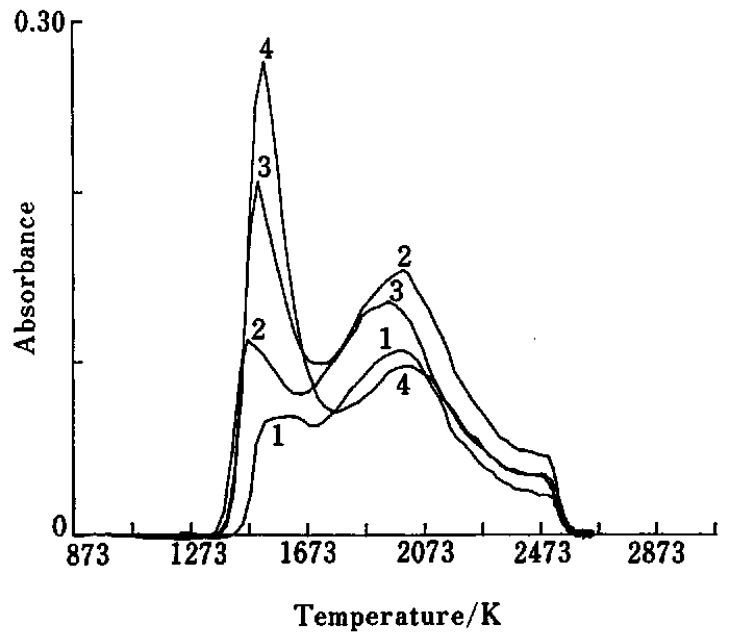

Fig. 6 Effect of the charring temperature on the atomic absorption signal of $10 \mathrm{ng}$ gallium using $0.5106 \%$ oxygen mixture. Charring temperature in K: 700 (1); 830 (2); 1030 (3); 1240 (4).

reactivity of the tube wall is expected. Figure 7 shows the effect of the purge gas flow rate, for a $0.7612 \%$ oxygen mixture, on the atomic absorption transient signal. $T_{\text {app }}$ was constant within the experimental error. While the integrated absorbance and the absorbance of the second and third peaks decrease, the absorbance of the first peak increases. The enhanced response of the first peak demonstrates an increase in the fraction of gallium atomized by the on-surface reduction (Eq. (6)) due to an increase in the extent of the oxidation of the tube wall, or in the number of active sites, during atomization.

\section{References}

1. P. V. Botha and J. Fazakas, Anal. Chim. Acta, 162, 413 (1984).

2. K. Nakamura, M. Fujimori, H. Tsuchiya and H. Orii, Anal. Chim. Acta, 138, 129 (1982).

3. X.-Q. Shan, Z.-N. Yuan and Z.-M. Ni, Anal. Chem., 57, 857 (1985).

4. X.-Q. Shan, Z.-M. Ni and Z.-N. Yuan, Anal. Chim. Acta, 171, 269 (1985).

5. J. C. Bailar, "Comprehensive Inorganic Chemistry", Pergamon Press, New York, 1973.

6. S. G. Salmon and J. A. Holcombe, Anal. Chem., 54, 630 (1982).

7. B. V. L'vov, Spectrochim. Acta, 33B, 153 (1978).

8. T. McAllister, J. Anal. At. Spectrom., 5, 171 (1990).

9. A. K. Gilmutdinov, Y. A. Zakharov, V. P. Ivanov and A.

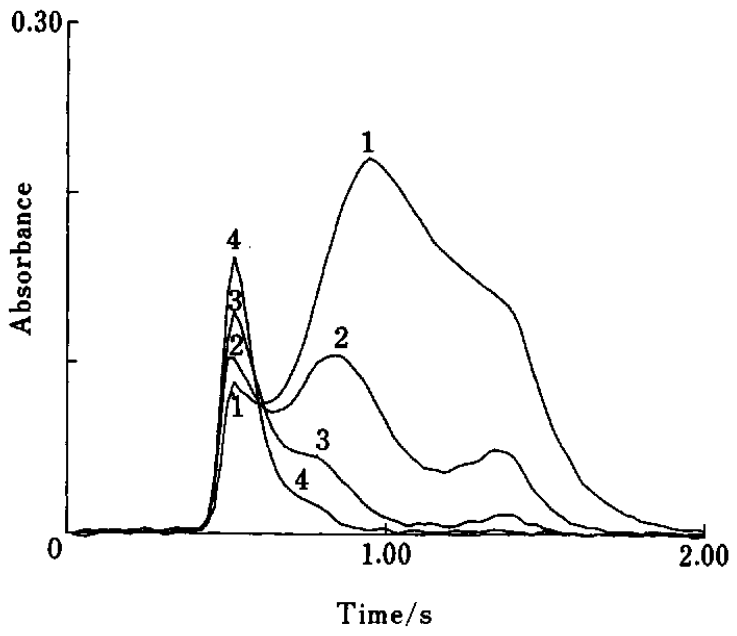

Fig. 7 Effect of inner gas flow rate on the atomic absorption signal of $10 \mathrm{ng}$ gallium using $0.7612 \%$ oxygen mixture. Inner gas flow rate in $\mathrm{ml} \mathrm{mim}^{-1}: 0$ (1); 30 (2); 60 (3); 90 (4).

V. Voloshin, J. Anal. At. Spectrom., 6, 505 (1991).

10. A. K. Gilmutdinov, Y. A. Zakharov, V. P. Ivanov, A. V. Voloshin and K. Dittrich, J. Anal. At. Spectrom., 7, 675 (1992).

11. K. Kuga, Bumseki Kagaku, 30, 529 (1981).

12. S. G. Salmon, R. H. Davis and J. A. Holcombe, Anal. Chem., 53, 324 (1981).

13. R. E. Sturgeon and S. S. Berman, Anal. Chem., 57, 1268 (1985).

14. B. V. L'vov and G. N. Ryabchuk, Spectrochim. Acta, 37B, 673 (1982).

15. S. Imai, K. Kosakai, Y. Nishiyama, K. Saito and Y. Hayashi, Anal. Sci., submitted.

16. S. Imai, K. Kosakai, Y. Nishiyama, K. Saito and Y. Hayashi, Anal. Sci., submitted.

17. F. J. Vastola, P. J. Hart and P. L. Walker Jr., Carbon, 2, 65 (1964).

18. N. R. Laine, F. J. Vastola and P. L. Walker Jr., J. Phys. Chem., 67, 2030 (1963).

19. R. E. Sturgeon, K. W. M. Siu and S. S. Berman, Spectrochim. Acta, 39B, 213 (1984).

20. G. F. R. Gilchrist, C. L. Chakrabarti and J. P. Byrne, J. Anal. At. Spectrom., 4, 533 (1989).

21. S. Imai and Y. Hayashi, Bull. Chem. Soc. Jpn., 65, 871 (1992).

22. R. P. Burns, J. Chem. Phys., 44, 3307 (1966).

23. R. C. West, "Handbook of Chemistry and Physics", 67th ed., CRC Press, Boca Raton, 1980.

(Received July 4, 1994)

(Accepted September 27, 1994) 